\title{
Identifying noisy and quiet modes in a jet
}

\author{
P. Jordan*, M. Schlegel ${ }^{\dagger}$, O. Stalnov ${ }^{\ddagger}$, B.R. Noack ${ }^{\dagger}$, and C.E. Tinney*
}

In the current jet noise study, an empirical modal decomposition is proposed which distills the noisy and quiet modes of the flow field. In particular, the POD of flows is generalised for an optimal resolution of the far-field noise as opposed to a least-order representation of the hydrodynamic fluctuation level. This decomposition technique, which we call 'most observable decomposition (MOD)', is based on a linear cause-effect relationship between the hydrodynamics (cause) and the far-field acoustics (observed effect). In the current study, this relationship is identified from a linear stochastic estimation between the flow field and the far-field pressure - taking into account the propagation time of sound. We employ MOD to turbulent jet noise at $M a=0.9, R e=3600$ using CFD/CAA data from RWTH Aachen. While more than 350 POD modes are necessary to capture only $50 \%$ of the flow fluctuation energy, a mere 24 MOD modes resolve $90 \%$ of the far-field acoustics. Evidently, far-field noise acts as filter which 'sees' only a low-dimensional subspace of the flow and 'ignores' silent subspaces which contain a large amount of fluctuation energy. The MOD methodology yields 'least-order' representations of any other observable as well assuming a linear relationship between flow and observable.

\section{Introduction}

At present there is no general consensus as to precisely which aspects of a free jet's flow dynamic are most important in the production of sound. There is of course a large body of experimental and numerical evidence for a number of 'hot-spots' in terms of sound production, for example: the close of the annular mixing-layer at the end of the potential core and the subsequent transition to self-similarity have been argued to be important by a number of researchers, ${ }^{1,2,3,4,5}$ violent intermittent entrainment of coherent structures into the high-velocity potential regions of the flow - also interpreted as the collapse of large-scale instabilities ${ }^{13,8}$ being shown to be important; quasi-irrotational wavy-wall-like mechanisms, probably most important in the region upstream of the close of the potential core; ${ }^{6}$ vortex pairing, again in regions upstream of the end of the potential core $;^{7}$ vortex eigen-oscillations associated with coherent structures $;^{10,11,12}$ incoherent fine-scale turbulence $;{ }^{9}$ instabilities in the near-nozzle region, possibly rendered efficient by the presence of the nozzle. ${ }^{14}$ However, the precise nature and relative contributions of each of these mechanisms are not well understood.

In this work a methodology is developed, based on modal decompositions of the flow and acoustic fields of a $\mathrm{M}=0.9, \mathrm{Re}=3600$ jet computed by an LES of the flow and CAA, based on APE and FWH formulae. ${ }^{15,16}$ This methodology aims to identify the most important flow events with respect to a number of observer points in the acoustic far-field. The technique involves monitoring the flow over linearly related observables measured in an $L_{2}$ norm. The least dimensional flow representation is then derived with respect to an observable subspace derived from the far-field pressure.

*Laboratoire d'Études Aérodynamiques, CNRS UMR 6609, Université de Poitiers, France

${ }^{\dagger}$ Institute of Fluid Dynamics and Technical Acoustics, Berlin University of Technology, Berlin, Germany

$\ddagger$ University of Tel Aviv, Israel 


\section{Outline of the technique}

Proper Orthogonal Decomposition (hereafter POD) is first used to obtain a Galerkin approximation of the flow field:

$$
\mathbf{u}^{[0 . . N]}(\mathbf{x}, t):=\sum_{i=0}^{N} a_{i}^{u}(t) \mathbf{u}_{i}(\mathbf{x}),
$$

where the mean field is termed $\mathbf{u}_{0}\left(a_{0}^{u}=1\right)$ and the POD modes $\mathbf{u}_{i}$ are spatial expansion modes. The POD modes are obtained by the POD snapshot method, which is based on a snapshot ensemble $\left\{\mathbf{u}^{i}\right\}_{i=1}^{M_{u}}$ of the flow field utilising the above mentioned LES. Two key elements in POD snapshot method ${ }^{17}$ are: the inner product of the velocity fluctuations,

$$
(\mathbf{u}, \mathbf{v})_{u}:=\int d \mathbf{x} \mathbf{u}(\mathbf{x}) \cdot \mathbf{v}(\mathbf{x})
$$

and the correlation matrix,

$$
R_{u}^{m n}=\frac{1}{M_{u}}\left(\mathbf{u}^{m}-\mathbf{u}_{0}, \mathbf{u}^{n}-\mathbf{u}_{0}\right)_{u},
$$

which comprises second-order structural information of the turbulence. The POD modes $\mathbf{u}_{i}$ decompose the flow field most efficiently for total kinetic energy (total fluctuation level $\frac{1}{M_{u}} \sum_{m=1}^{M_{u}} R_{u}^{m m}$ ), and are obtained by means of a diagonalisation of the correlation matrix; and the inner-product determines the optimality of the modal decomposition. This is an important point which forms the basis of the technique presented in this work. It is worth noting that this first step does not necessarily need to be restricted to the velocity field: a flow quantity such as an acoustic-analogy source term can alternatively be used, in which case a Galerkin approximation of the source dynamics is obtained. ${ }^{25}$ Where the approach described in this paper is concerned such a choice may present considerable advantages, as the relationship between such source terms (Lighthill for example) and the radiated sound pressure is generally a linear one. The downside of such an approach is that an evolution equation is not readily available for acoustic-analogy source-terms, and thus a straight forward low-order dynamical-system identification (LODS) is not feasible.

The radiated pressure field, sampled on a line array at 30 diameters from and parallel to the jet axis, is similarly represented by its Galerkin approximation:

$$
\mathbf{p}^{\left[0 . . N_{p}\right]}(\mathbf{x}, t):=\sum_{i=0}^{N_{p}} a_{i}^{p}(t) \mathbf{p}_{i}(\mathbf{x}),
$$

which is also obtained by POD snapshot method, using the inner product

$$
(p, q)_{p}:=\left.\int d x p(r, \theta, x) q(r, \theta, x)\right|_{r=30 D, \theta=0^{\circ}},
$$

and the correlation matrix

$$
R_{p}^{m n}=\frac{1}{M_{p}}\left(p^{m}-p_{0}, p^{n}-p_{0}\right)_{p}
$$

based on the far-field snapshot ensemble $\left\{p^{i}\right\}_{i=1}^{M_{p}}$. Here, the POD modes $p_{i}$ decompose the far-field most efficiently for the total level of far-field fluctuation $\frac{1}{M_{p}} \sum_{m=1}^{M_{p}} R_{p}^{m m}$.

The highly-complex dynamics of the flow, and the somewhat less-complex dynamics of the acoustic pressure field are thus characterised by the POD modes $\mathbf{u}_{i}(\mathbf{x})$ and $\mathbf{p}_{i}(\mathbf{x})$, and the Fourier coefficients $a_{i}^{u}(t)$ and $a_{i}^{p}(t)$ : the Fourier coefficients animate the eigenfunctions. The POD is shown schematically in figure 1.

We wish to understand the causal relationship which translates the physical mechanisms by which the flow dynamics $\left(\sum_{i=0}^{N} a_{i}^{u}(t) \mathbf{u}_{i}(\mathbf{x})\right)$ drive the far-field $\left(\sum_{i=0}^{N_{p}} a_{i}^{p}(t) \mathbf{p}_{i}(\mathbf{x})\right)$. In a first stage we assume a linear relationship between the fluctuating turbulent velocity and the acoustic far-field pressure: there is a considerable

2 of 9

American Institute of Aeronautics and Astronautics 

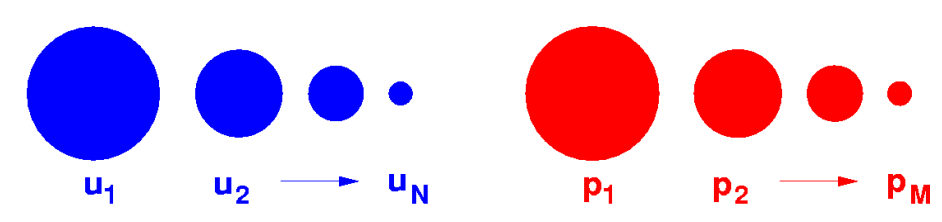

Figure 1. Schematic representation of POD modes: size of circles represents the level of resolved fluctuation level of each mode; in blue: velocity modes, optimal resolution of total kinetic energy based on the inner-product $(u, v){ }_{u}$; in red: pressure modes, optimal resolution of total far-field fluctuation level based on the inner-product $(p, q)_{p}$.

body of evidence to suggest that such an approximation is reasonable: the fast-pressure term (sometimes referred to as 'shear-noise') has been shown to dominate in free-jets in terms of the hydrodynamic, turbulent pressures ${ }^{26}$ and to correlate better with the far-field pressure than the quadratic slow-pressure ('selfnoise'); ${ }^{18,21,24,22,23,19,1,20,29}$ Coiffet et al. ${ }^{6}$ have furthermore demonstrated that the coherent flow structures generate sound via a linear mechanism in the region upstream of the end of the potential core, and Guitton et al. ${ }^{30}$ have shown that such a mechanism exists as far downstream as 10D, and for Mach number ranging from 0.15 to 0.6: we therefore perform a linear mapping between the POD mode coefficients of the velocity field and those of the pressure field:

$$
a_{i}^{p}(t)=\sum_{j=1}^{N} C_{i j}^{p u} a_{j}^{u}(t-\tau),
$$

where $\tau$ represents the time delay of acoustic propagation and $C_{i j}^{p u}$-which embodies the degree of linearity between the dynamics of the turbulent velocity field and the acoustic far-field (via their Fourier coefficients) is obtained using Linear Stochastic Estimation.

The role of the operator $\mathbf{C}$, given by the matrix elements $C_{i j}^{p u}$, can be understood in a number of ways: it provides a means of stripping the highly complex turbulence dynamics down to the components which were essential in linearly driving the acoustic far-field; as such, it amounts to a filtering operation which identifies the radiating jet dynamics (very similar to the action of a Green's function): convolving $\mathbf{C}$ with the velocity field $\left(a^{u}\right)$ gives the far-field pressure; convolving its pseudo-inverse, $\mathbf{C}^{-}$, with the pressure field $\left(a^{p}\right)$ identifies the turbulence dynamics which were linearly implicated in the production of sound, or in other words, linearly observable from the far-field (see figure 2: blue circles represent the observable jet dynamics); it allows us to study the jet dynamics in a linear observer sub-space which has considerably fewer degrees of freedom than the complete turbulence field. These ideas are illustrated schematically in figures 2. For a particular pseudo-inverse, which minimises the energy of the image, this procedure coincides with the method of extended POD. ${ }^{31,32}$

In figure 3 the blue phase-diagrams represent (very simplified) model jet dynamics, in two and three dimensions; the green lines represent linear mappings, which define the projections of the higher-dimensional jet dynamics onto the linear subspaces (shown in red), as seen by a single observer in the $2 \mathrm{D}$ case, and by two observers in the 3D case. In order to discern the complexity of the dynamics in the linear subspace a Proper Orthogonal Decomposition could be performed; however, in such a situation, because the inner product of the problem is defined using the velocity field, such a decomposition is optimal in terms of the kinetic energy of the filtered velocity field. We are interested in a decomposition which is optimal in terms of the sound energy radiated by the flow, and so we redefine the inner product as follows:

$$
(\mathbf{u}, \mathbf{v})_{*}:=(\mathbf{C} \mathbf{u}, \mathbf{C} \mathbf{v})_{p}=\sum_{i=1}^{S}\left(\sum_{j=1}^{N} C_{i j}^{p u} a_{j}^{u}\right)\left(\sum_{j=1}^{N} C_{i j}^{p u} a_{j}^{v}\right)
$$




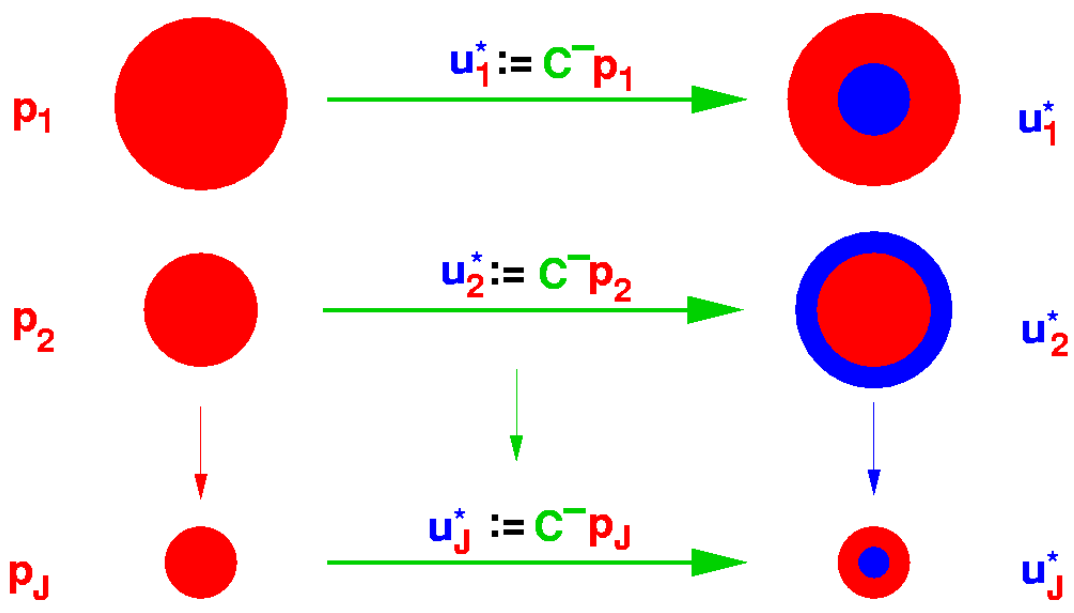

Figure 2. Schematic representation of MOD: acoustically optimised Galerkin representation of jet's 'causal' linearsubspace. These linear subspaces are determined to be the pseudo-inverse images of the far-field POD mode. Blue and red circles are explained like in figure 1. The property of optimal far-field fluctuation resolution is maintained for a sufficiently accurate cause-effect relationship (7) of hydrodynamics and aeroacoustics.
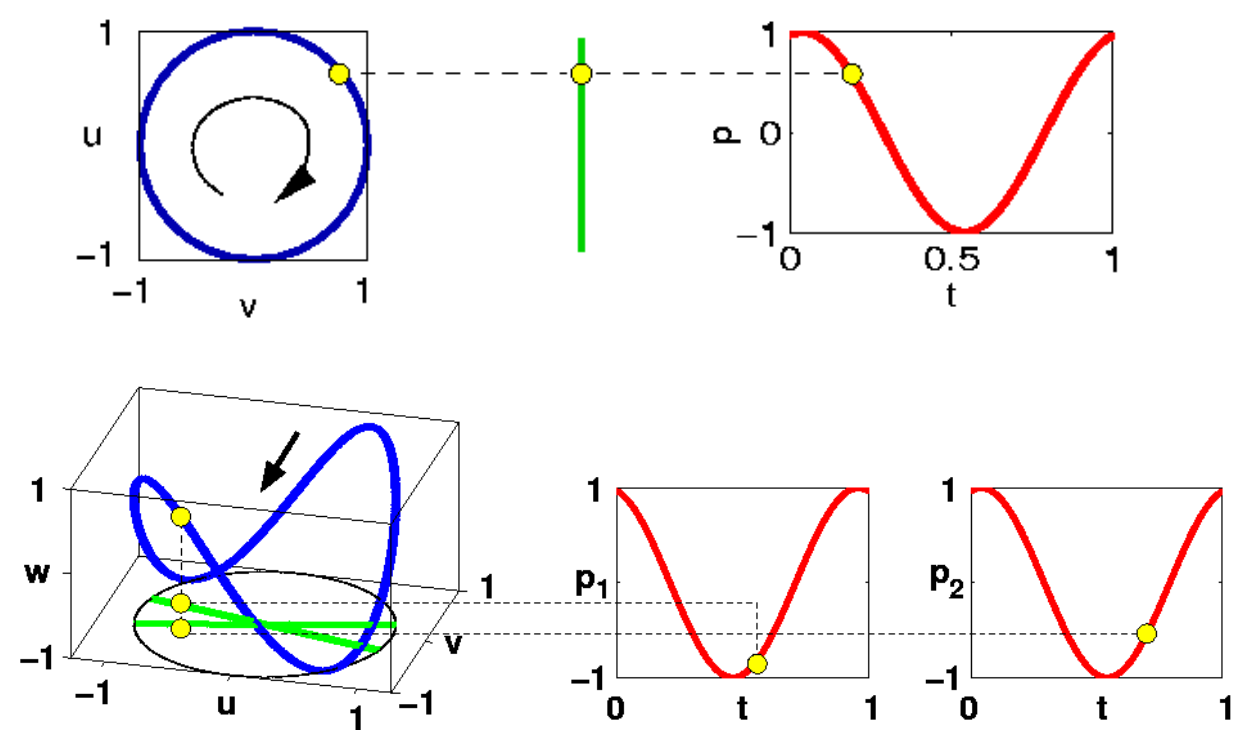

Figure 3. Two- and three-dimensional schematic representations of the link (via linear mapping: shown in green) between complex high-dimensional jet dynamics (in blue) and the less-complex, lower-dimensional acoustic dynamics (in red). In the two-dimensional representation on the top row, the (red) far-field signal $p=\cos 2 \pi t(t \in[0,1])$ can be observed by the projection $p=\mathbf{C} \mathbf{u}$ of the (blue) field $\mathbf{u}=(\cos 2 \pi t, \sin 2 \pi t)^{T}$ onto the linear subspace $\mathbf{C}=(1,0)($ green line). In the three dimensional representation, the (red) far-field signals $p_{1}=\sqrt{\frac{15}{16}} \cos 2 \pi t-\frac{1}{4} \sin 2 \pi t$ and $p_{2}=\sqrt{\frac{15}{16}} \cos 2 \pi t+\frac{1}{4} \sin 2 \pi t$ can be observed by the projection $\left(p_{1}, p_{2}\right)^{T}=\mathbf{C u}$ of the (blue) field $\mathbf{u}=(\cos 2 \pi t, \sin 2 \pi t, \sin 4 \pi t)^{T}$ onto the linear subspaces represented by the rows of $\mathbf{C}=\left(\mathbf{C}_{1}, \mathbf{C}_{2}\right)^{T}$ with $\mathbf{C}_{1}=\left(\sqrt{\frac{15}{16}},-\frac{1}{4}\right)$ and $\mathbf{C}_{2}=\left(\sqrt{\frac{15}{16}}, \frac{1}{4}\right)$ respectively $($ green lines) 
The POD problem is thus posed such that: (1) the kernel of the problem comprises two-point correlations of the filtered velocity field, and so velocity modes will be obtained; but, (2) the modal decomposition is optimal with respect to the sound energy radiated from the flow. This is the MOD principle, and it is shown schematically in figure 4 .

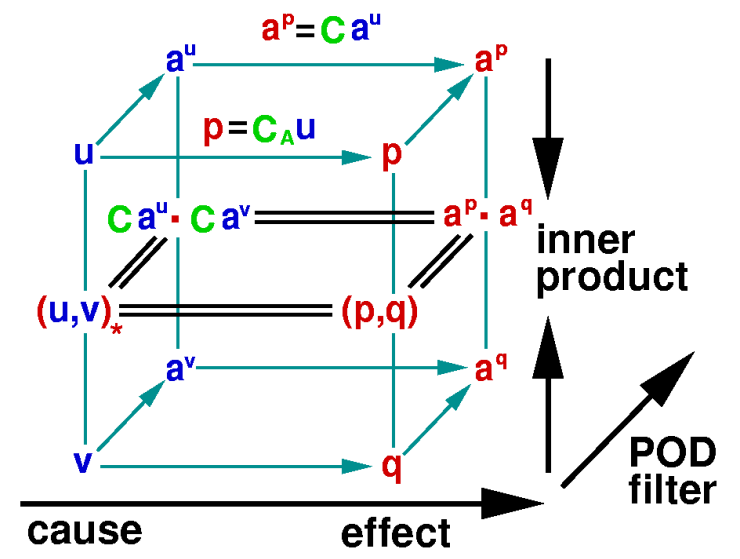

Figure 4. Schematic representation of MOD idea: cause (velocity, blue) and effect (pressure, red): left and right-hand vertical faces of cube; POD filter (Galerkin representations of dynamics): front and back vertical faces of cube; cause and effect related by linear-mapping (green): this is the key to identifying the jet's 'causal' subspace; identity between inner-products (middle horizontal section) is the key to identifying loud 'causal' modes, i.e. MOD modes. This figure is explained in more detail below.

The left and right faces of the cube represent, respectively, the causal jet dynamics in terms of its velocity field $\boldsymbol{u}(\boldsymbol{v})$ and their effect, the far-field pressure $\boldsymbol{p}(\boldsymbol{q})$ : they are related by the linear mapping $\boldsymbol{C}$. Moving from the front face of the cube to the back face we change from a classical space-time description of the two fields to Galerkin approximations of these by means of a POD filter (dynamics now captured by the Fourier coefficients $\boldsymbol{a}^{\boldsymbol{u}}, \boldsymbol{a}^{\boldsymbol{v}}, \boldsymbol{a}^{\boldsymbol{p}}$ and $\boldsymbol{a}^{\boldsymbol{q}}$ ). The central horizontal plane contains the inner-products, and it is here that we find the key to the MOD: a velocity inner-product is defined which is equal to the inner-product of the far-field pressure: this forces an optimisation of the jet dynamics - which are here filtered by the linear mapping - in terms of the far-field pressure when the POD filter is performed, and allows thence an identification of the most linearly observable velocity modes.

\section{Results}

The first important result is manifest in the MOD convergence: this is shown in figure 5, where it is compared with the convergence of the POD. While more than 350 POD modes are necessary to capture $50 \%$ of the TKE of the jet, $90 \%$ of the radiated sound energy is captured with only 24 MOD modes. This is a testament to the significantly lower-dimensional nature of the sound-producing jet dynamics: such reductions in complexity were also observed, respectively, by Freund ${ }^{27}$ and by Cabana et al., ${ }^{28}$ when they isolated the radiating component of the Lighthill source term: in a low Reynolds-number jet (very similar to the one studied here), and in a two-dimensional temporal mixing-layer. It should be emphasised that this reduced complexity of the sound-producing jet dynamics is good news for control: while the complexity of a turbulent jet presently precludes the direct application of control theory, the existence of such a loworder sound-producing component will be conducive to the identification of a dynamical-system, a dynamic observer and an efficient control law.

A second interesting result is manifest - with respect to the physical mechanisms which underlie the production of sound in this low Reynolds-number flow - in the first six MOD modes, shown in figure 6. The higher MOD modes all show variously disorganised, smaller-scale activity and are not shown here. It can 


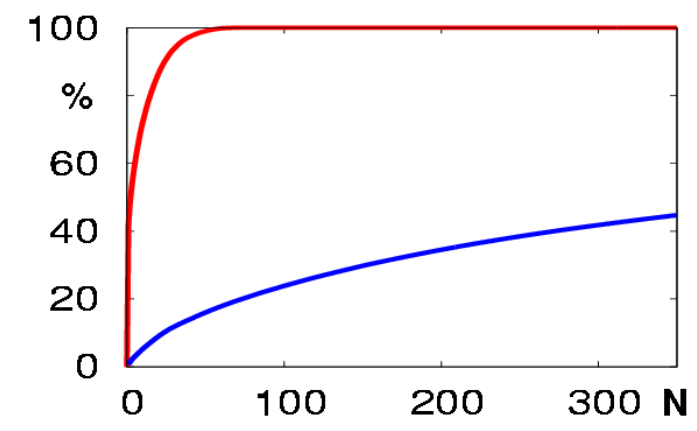

Figure 5. Convergence of POD and MOD: The resolution of total kinetic energy by $N$ POD modes is displayed in percentages over $N$ (blue line). The resolution of total far-field fluctuation level by $N$ MOD modes is displayed in percentages over $N$ (red line).

be seen how the MOD modes translate a number of different characteristic flow features and events. The first two, which are the most energetic modes in terms of the far-field pressure (resolving about $48 \%$ of the far-field), reveal asymmetric (helical-looking) streaks in the region just downstream of the end of the potential core. This is in qualitative agreement with experiments such as those of Juve et al, ${ }^{1}$ Guj et al. ${ }^{2}$ and Hileman et al. ${ }^{4}$ The next three most energetic modes (which contribute about $12 \%$ of the far-field) show structures comprising highly coherent, axisymmetric vortex-ring-like structures in the region upstream of the end of the potential core. This resembles the wavy-structure of the radiating component of the Lighthill source term, as identified by Freund, ${ }^{27}$ and is in excellent agreement with the linear wavy-wall mechanism identified experimentally by Coiffet et al. ${ }^{6}$ While the higher order modes (which account for $40 \%$ of the far-field) reveal a more disorganised structure, mainly downstream of the end of the potential core, they remain relatively structured: mode six shows the remnants of the axisymmetric flow-structures, particularly in the v-component.

In summary: the sound producing jet dynamics — as identified by MOD in this low Reynolds-number flow - are dominated by convected axisymmetric vortex-rings in the region upstream of the end of the potential core. Their non-negligible contribution to the far-field (12\%) is an interesting result; and one which is contrary to: the school-of-thought which holds that this mechanism is inefficient on account of its subsonic convection velocity; and, the argument that such mechanisms require non-linear mode interactions to become efficient in the production of sound. (remember a linear relationship has been assumed here between the velocity fluctuations and the far-field; a similar linear mechanisms is demonstrated by Coiffet et al.; ${ }^{6}$ and, another strongly linear coupling between hydrodynamic pressure and velocity is proved by Noack et al. ${ }^{33}$ ). At the end of the potential core these axisymmetric structures are destroyed and transformed into streak-like structures (visual inspection reveals an azimuthal mode 5 or 6 ): this is the dominant sound-producing event in this flow, contributing of the order of $48 \%$, and it is in agreement with a large body of experimental and numerical evidence.

\section{Conclusion}

A method which we call the Most Observable Decomposition (MOD) has been developed for identifying the sound-producing dynamics of a turbulent jet. Application to numerically computed low Reynolds number jet has led to the following observations: (1) while over 350 POD modes are required to capture $50 \%$ of the TKE, $90 \%$ of the sound energy is capture with only 24 MOD; (2) the MOD mode demonstrate how over $48 \%$ of the sound generated by this flow is due to the destruction of coherent structures in the transition region, downstream of the end of the potential core; (3) over $12 \%$ of the sound production is found to be 

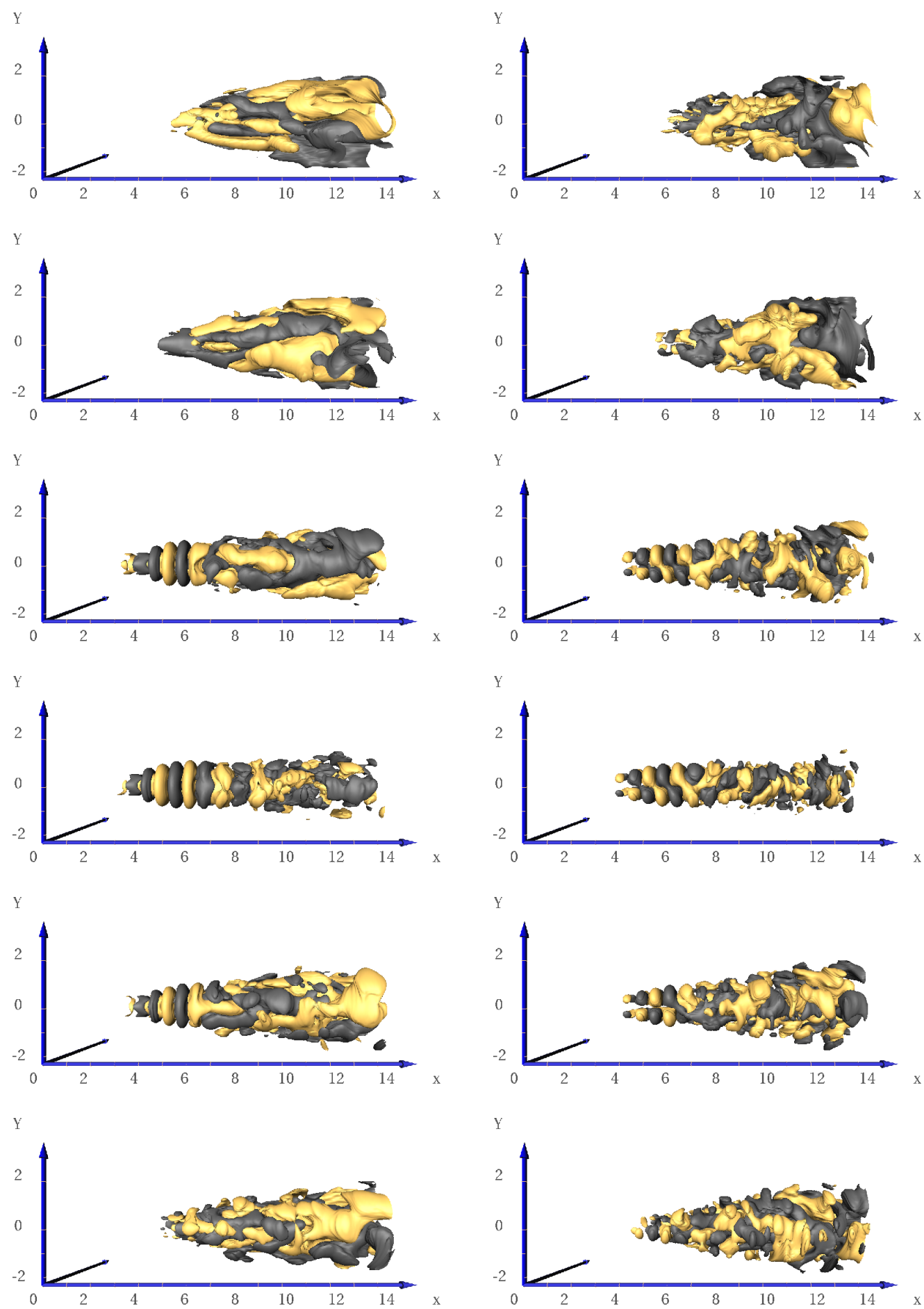

Figure 6. First six (1 to 6 from top to bottom) 3D MOD modes: left column: u-component; right column: radial component. Isosurfaces of positive (yellow) and negative (dark) values are shown. 
related to convected axisymmetric vortex-rings, whose signature persists into the transition region. Further analysis of this data is ongoing

\section{Acknowledgements}

Part of this work was performed during the second European Forum on Flow Control, which was supported by AIRBUS through the CAFEDA research program, and which took place at the LABORATOIRE D'Études AÉrodynamique, Poitiers from May to July 2006. Support of the Deutsche Forschungsgemeinschaft (DFG) under grants NO 258/1-1, NO 258/2-3 and the SFB 557 is acknowledged. The authors also gratefully acknowledge the Agence Nationale de la Recherche (ANR) for financial support. We thank Boye Ahlborn, Jean-Paul Bonnet, Joël Delville, Pierre Comte, Bill George, Hans-Christian Hege, Mark Pastoor, Gilead Tadmor, Jon Scouten, Tino Weinkauf, Elmar Gröschel, Maja Wänström and Wolfgang Schröder for inspiring and helful discussion. The 3D figures have been prepared with Amira.

\section{References}

${ }^{1}$ D. Juvé, M. Sunyach, G. Comte-Bellot. Intermittency of the noise emission in subsonic cold jets. J. Sound Vib., 71(3), 1980.

${ }^{2}$ G. Guj , C. Carley, R. Camussi. Acoustic identification of coherent structures in a turbulent jet. J. Sound Vib., 259(5), 1037-1065, 2003.

${ }^{3}$ C. Bogey, C. Bailly, D. Juvé. Noise investigation of a high subsonic, moderate Reynolds number jet using a compressible large eddy simulation. Theoret. Comput. Fluid Dyn., 16, 2003.

${ }^{4}$ J. Hileman, E. Caraballo, B. Thurow, M. Samimy. Differences in dynamics of an ideally expanded Mach 1.3 jet during noise generation and relative quiet periods. 10th AIAA/CEAS Aeroacoustics conference, paper AIAA-2004-3015, Manchester, May 2004.

${ }^{5}$ J.H. Citriniti \& W.K. George. Reconstruction of the global velocity field in the axisymmetric mixing-layer utilizing the proper orthogonal decomposition. J. Fluid Mech. 418:137-166

${ }^{6}$ F. Coiffet, P. Jordan, J. Delville, Y. Gervais, \& F. Ricaud. Coherent structures in subsonic jets: a quasi-irrotational source mechanism?. Int. J. Aeroacoustics, 5(1), 2006.

${ }^{7}$ J. Laufer, T.-C. Yen. Noise generation by a low mach number jet. J. Fluid Mech., 154, 1983.

${ }^{8}$ K. Viswanathan, M.L. Shur, P.R. Spalart, M. Kh. Strelets. Computation of the flow and noise of round and beveled nozzles. 12th AIAA/CEAS Aeroacoustics conference, paper-2006-2445, Cambridge, May 2006.

${ }^{9}$ C. Tam, M. Golebiowski, J.M. Seiner. On the two components of turbulent mixing noise from supersonic jets. AIAA paper, 96-1716, May 1996.

${ }^{10}$ V.F. Kopiev, S.A. Chernyshev. Vortex ring eigen-oscillations as a source of sound. J. Fluid Mech., 341:19-57, 1997.

${ }^{11}$ V.F. Kopiev, M. Zaitsev, S.A. Chernyshev. Comparison of vortex ring noise theory with excited jet acoustic measurements. 10th CEAS-ASC Workshop, Trinity College, Dublin, Ireland, 2006.

${ }^{12}$ V.F. Kopiev, M. Zaitsev, S.A. Chernyshev, A.N. Kotova. The role of large-scale vortex in a turbulent jet noise. AIAA $P a-$ per, 99-1839, 1999.

${ }^{13}$ J.L. Stromberg, D.K. McLaughlin, T.R. Troutt. Flow field and acoustic properties of a Mach number 0.9 jet at a low Reynolds number. J. Sound Vib., 72(2):159-176, 1980.

${ }^{14}$ P. Jordan, C.E. Tinney. A study in the near pressure field of co-axial subsonic jets. Under consideration for publication in J. Fluid Mech., 2007.

${ }^{15}$ E. Gröschel, W. Schröder, M. Schlegel, J. Scouten, B.R. Noack, P. Comte. Reduced-order representation of turbulent jet flow and its noise source. CEMRACS 2005, published in: ESAIM Proceedings, Vol. 16, 33-50, 2007

${ }^{16}$ R. Ewert, W. Schröder. Acoustic pertubation equations based on flow decomposition via source filtering. J. Comput. Physics, 188:365-398, 2003.

${ }^{17}$ L. Sirovich. Turbulence and the dynamics of coherent structures, Part I: Coherent structures. Quart. Appl. Math., XLV, 561-571, 1987.

${ }^{18}$ H.K. Lee, H.S. Ribner. Direct correlation of noise and flow of a jet. J. Acoust. Soc. Amer., 52(5), 1972.

${ }^{19} \mathrm{M}$. Schaffer. Direct measurements of the correlation between axial in-jet velocity fluctuations and far field noise near the axis of a cold jet. J. Sound Vib., 64(1), 73-83, 1979.

${ }^{20}$ M. Schaffer, J.P. Hancy. Investigation of the noise emitting zones of a cold jet via causality correlations. J. Sound Vib., 81(3), 377-391, 1982.

${ }^{21}$ T.D. Scharton, P.H. White. Simple pressure source model of jet noise. J. Acoust. Soc. Amer., 52(1), 1972.

8 of 9

American Institute of Aeronautics and Astronautics 
22 J.M. Seiner. The distribution of jet source strength intensity by means of a direct correlation technique. PhD thesis, Pennsylvania State University, 1974.

${ }^{23}$ J.M. Seiner, G. Reethof. On the distribution of source coherency in subsonic jets. AIAA Paper, 74-4, Washington, 1974.

${ }^{24}$ T.E. Siddon, R. Rackl. Cross-correlation analysis of flow noise with fluid dilatations as source fluctuation. J. Acoust Soc. Amer., 51, 96(A), 1972.

${ }^{25}$ M. Schlegel, B.R. Noack, E. Gröschel, W. Schröder, P. Comte, P. Jordan. Reduced-Order Analysis of Turbulent Jet Noise. Proceedings of the DAGA'07 conference, 2007.

${ }^{26}$ C.E. Tinney, P. Jordan, A. Hall, J. Delville, M.N. Glauser. A time-resolved estimate of the turbulence and sound source mechanism in a subsonic jet flow. J. Turbulence, 8(1):1-20, 2006.

${ }^{27}$ J.B. Freund. Noise sources in a low-Reynolds-number turbulent jet at Mach 0.9. J. Fluid Mech., 438:277-305, 2001.

${ }^{28}$ M. Cabana, V. Fortuné, P. Jordan. A look inside the Lighthill source term., Submitted to Theoret. Comput. Fluid Dyn., 2007.

${ }^{29}$ J. Panda, R.G. Seasholtz, K.A. Elam. Investigation of noise sources in high-speed jets via correlation measurements. J. Fluid Mech., 537:349-385, 2005.

${ }^{30}$ A. Guitton, E. Laurendeau, P. Jordan, J. Delville. Velocity dependence of the near pressure field of subsonic jets., AIAA-2007-3661, 2007.

${ }^{31}$ J. Borée. Extended proper orthogonal decomposition: a tool to analyse correlated events in turbulent flows. Exp. Fluids, $35,188,2003$.

${ }^{32}$ C. Hoarau, J. Borée, J. Laumonier, Y. Gervais. Analysis of the wall pressure trace downstream of a separated region using extended proper orthogonal decomposition. Phys. Fluids, 18, 055107, 2006.

${ }^{33}$ B.R. Noack, P. Papas, P.A. Monkewitz. The need for a pressure-term representation in empirical Galerkin models of incompressible shear flow. J. Fluid Mech. 523, 339-365, 2005. 